\title{
Status of AKK Fragmentation Functions
}

\author{
Bernd A. Kniehl \\ II. Institut für Theoretische Physik, Universität Hamburg, \\ Luruper Chaussee 149, 22761 Hamburg, Germany
}

\begin{abstract}
We summarize the improvements to the previous AKK extraction of fragmentation functions for $\pi^{ \pm}, K^{ \pm}, p / \bar{p}, K_{S}^{0}$ and $\Lambda / \bar{\Lambda}$ hadrons at next-to-leading order implemented in the AKK08 sets.
\end{abstract}

\section{Introduction}

In the framework of the QCD-improved parton model, the inclusive production of single hadrons is described by means of fragmentation functions (FFs) $D_{a}^{h}\left(x, \mu^{2}\right)$. At leading order, the value of $D_{a}^{h}\left(x, \mu^{2}\right)$ corresponds to the probability for the parton $a$ produced at short distance $1 / \mu$ to form a jet that includes the hadron $h$ carrying the fraction $x$ of the longitudinal momentum of $a$. Unfortunately, it is not yet possible to calculate the FFs from first principles, in particular for hadrons with masses smaller than or comparable to the asymptotic scale parameter $\Lambda$. However, given their $x$ dependence at some energy scale $\mu$, the evolution with $\mu$ may be computed perturbatively in QCD using the timelike Dokshitzer-Gribov-Lipatov-Altarelli-Parisi (DGLAP) equations 2. This allows us to test QCD quantitatively within one experiment observing single hadrons at different values of center-of-mass energy $\sqrt{s}$ (in the case of $e^{+} e^{-}$annihilation) or transverse momentum $p_{T}$ (in the case of scattering). Moreover, the factorization theorem guarantees that the $D_{a}^{h}\left(x, \mu^{2}\right)$ functions are independent of the process in which they have been determined and represent a universal property of $h$. This enables us to make quantitative predictions for other types of experiments as well.

Our previous determinations of light-hadron FFs, yielding the BKK [3], KKP [4], and AKK [5] sets, were based entirely on $e^{+} e^{-}$annihilation data in the large- $x$ range, $x>0.1$, where $x=2 E_{h} / \sqrt{s}$ is the scaled hadron energy. In this presentation, we summarize the improvements from new theoretical input and additional experimental data implemented in the 2008 update of the AKK sets for charged pions, charged and neutral kaons, (anti)protons, and (anti)lambdas (AKK08) [6]. In Secs. 2 and 3, we discuss the most important improvements due to new theoretical and experimental inputs, respectively. In Sec. 团 we briefly mention how the AKK08 FFs compare with the AKK ones and those recently presented by Hirai, Kumano, Nagai, and Sudoh (HKNS) 7 and by de Florian, Sassot and Stratmann (DSS) 8]. In Sec. 5, we present an outlook.

\section{Theoretical improvements}

We incorporate hadron mass effects 9 , and fit the hadron mass in the case of the $e^{+} e^{-}$ analysis, which also has the effects of subtracting out other low- $\sqrt{s}$ and small- $x$ effects beyond the fixed-order approach, such as higher-twist, small- $x$ logarithms, etc. The fitted hadron masses are presented in Table 1, where they are compared with the true values. In the case of the baryons, the fitted masses are about $1 \%$ above the true values, which is consistent with scenarios in which the baryons are produced mainly from direct partonic fragmentation with a small contribution from decays from slightly heavier resonances. 


\begin{tabular}{|l|r|r|}
\hline Particle & Fitted & True \\
\hline$\pi^{ \pm}$ & 154.6 & 139.6 \\
$K^{ \pm}$ & 337.0 & 493.7 \\
$p / \bar{p}$ & 948.8 & 938.3 \\
$K_{S}^{0}$ & 343.0 & 497.6 \\
$\Lambda / \bar{\Lambda}$ & 1127.0 & 1115.7 \\
\hline
\end{tabular}

Table 1: Values of hadron masses (in $\mathrm{MeV}$ ) resulting from the charge-signunidentified AKK08 6] fits of $e^{+} e^{-}$ annihilation data and true values.

A greater excess is found for the pion mass, suggesting contributions to the sample from decays of heavier particles such as $\rho(770)$. The charged- and neutral-kaon masses are significantly below their true values. A possible explanation for this is that there are significant contributions from complicated decay channels, such that the direct partonic fragmentation approach is insufficient. For this reason we do not impose $\mathrm{SU}(2)$ isospin symmetry of $u$ and $d$ quarks between charged and neutral kaons, which we do for pions. In Fig. 1] the TPC [10] and TOPAZ [1] measurements of $(1 / \sigma)(\mathrm{d} \sigma / \mathrm{d} x)\left(e^{+} e^{-} \rightarrow p / \bar{p}+X\right)$ are compared with the calculations using the AKK08, AKK, HKNS, and DSS FFs. We observe that, owing to the inclusion of hadron-mass effects, the AKK08 set leads to the best description of both data sets in the low- $x$ range.
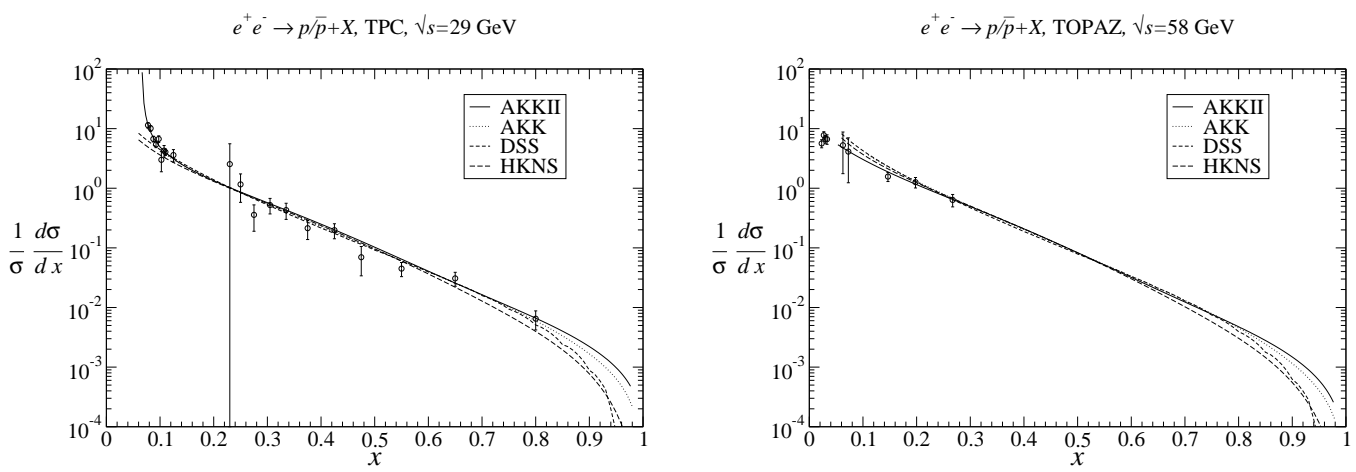

Figure 1: TPC [10] and TOPAZ [1] measurements of $(1 / \sigma)(\mathrm{d} \sigma / \mathrm{d} x)\left(e^{+} e^{-} \rightarrow p / \bar{p}+X\right)$ compared with AKK08 [6], AKK [5], HKNS [7, and DSS [8] results.

\begin{tabular}{|l|r|r|}
\hline Particle & Default & Unres. \\
\hline$\pi^{ \pm}$ & 519.7 & 520.8 \\
$K^{ \pm}$ & 417.3 & 488.2 \\
$p / \bar{p}$ & 525.1 & 538.0 \\
$K_{S}^{0}$ & 318.6 & 318.8 \\
$\Lambda / \bar{\Lambda}$ & 272.3 & 326.0 \\
\hline
\end{tabular}

Table 2: Minimized $\chi^{2}$ values in the charge-sign unidentified AKK08 [6] fits and analogous results without large- $x$ resummation.
We implement large- $x$ resummation in the quark coefficient function of $e^{+} e^{-}$reactions using the results from Ref. [12, since this is a simple improvement which modifies the cross section over the whole range in $x$ that we constrain. Large- $x$ resummation is also implemented in the DGLAP evolution of the FFs [13. As may be seen from Table 2, this results in a significant improvement in the fit for charged kaons, (anti)protons and (anti)lambdas, while $\chi^{2}$ is essentially unchanged for charged pions and neutral kaons. In Fig. 2, the TASSO [14] and OPAL [15] measurements of $(1 / \sigma)(\mathrm{d} \sigma / \mathrm{d} x)\left(e^{+} e^{-} \rightarrow \pi^{ \pm}+X\right)$ are compared with the default AKK08 6] results for the choices $k=M_{f}^{2} / s=1 / 4,1,4$ of factorization scale and with the analogous results without large- $x$ resummation. We observe that large- $x$ resummation improves the description of the experimental data in the region of intermediate to large $x$ values. 

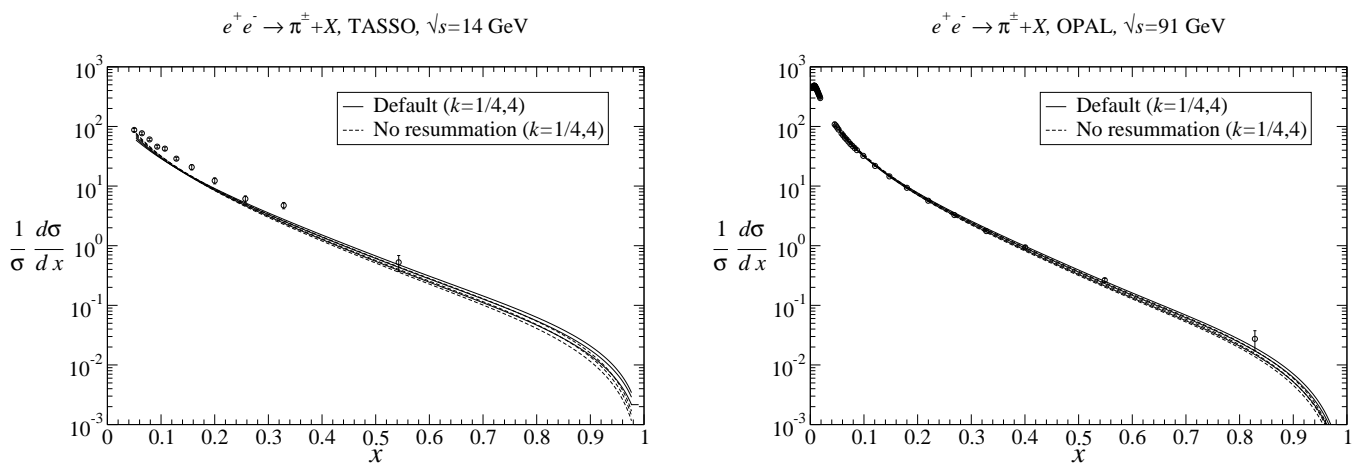

Figure 2: TASSO 14 and OPAL 15 measurements of $(1 / \sigma)(\mathrm{d} \sigma / \mathrm{d} x)\left(e^{+} e^{-} \rightarrow \pi^{ \pm}+X\right)$ compared with default AKK08 [6] results for scale choice $k=M_{f}^{2} / s=1 / 4,1,4$ and with the analogous results without large- $x$ resummation.

\section{Additional experimental input}

In addition to the constraints of the previous AKK fit, we impose further constraints on the charge-sign-unidentified FFs from the data for single inclusive production of identified particles $\left(p_{T} \geq 2 \mathrm{GeV}\right)$ from RHIC [16] the Tevatron [17, and $e^{+} e^{-}$reactions below the $Z$-boson pole and in the range $0.05<x<0.1$. While the untagged measurements from $e^{+} e^{-}$ reactions provide excellent constraints for the sums of the charge-sign unidentified FFs for quarks of the same electroweak charges, they do not constrain the remaining degrees of freedom at all. As in the previous AKK fit, these were constrained using quark-tagged data, while, in the new AKK08 fit, additional constraints are provided by the data from RHIC. These data are also much more sensitive to gluon fragmentation and impose (exclusively) new constraints on the charge-sign asymmetry FFs. Normalization errors were treated as systematic effects, i.e. these errors were incorporated via a correlation matrix. Their weights were fitted analytically and independently of the fit in order to further ascertain the quality of the fit, and their magnitudes were typically found to lie in the reasonable range of $0-2$.

While the results for the fitted masses suggest that the baryons are the best candidates for studying direct partonic fragmentation, there unfortunately exist some inconsistencies between the calculation and the measurements of the inclusive production of these particles at RHIC: The description of the STAR data for $\Lambda / \bar{\Lambda}$ fails, while the contribution from the initial protons' valence $d$ quarks to the charge-sign asymmetry for $p / \bar{p}$ from STAR is negative. Furthermore, while the contributions from the valence and sea-quark components of the initial protons to the cross section for charge-sign unidentified particle production at RHIC exhibit the expected behavior, the contribution from the valence $d$-quark component to the charge-sign asymmetry in (anti)proton production is negative. All these issues would be better understood in the context of an error analysis of the FFs.

\section{Comparisons with other FF determinations}

Comparing our FF sets to the recent HKNS 7 and DSS [8] ones, we typically find reasonable agreement for favored FFs, but not for unfavored ones, and large discrepancies exist at large 
$x$ in some cases (see Fig. 1).

\section{Outlook}

Future hadron-identified data from BABAR, CLEO, HERA, and RHIC will help to clarify the open issues mentioned above and significantly reduce the large uncertainties in much of the FF degrees of freedom. As for the analysis of high- $Q^{2}$ electroproduction data from HERA, hadron species and charge identification would be very useful.

\section{Acknowledgments}

The author thanks S. Albino and G. Kramer for the collaboration on the work presented here. This work was supported in part by the Deutsche Forschungsgemeinschaft through Grant No. KN 365/5-1 and by the Bundesministerium für Bildung und Forschung through Grant No. 05 HT6GUA.

\section{References}

[1] Slides: http: //indico. cern. ch/contributionDisplay $\cdot$ py? contribId=198\&sessionId=13\&conf Id=24657

[2] V.N. Gribov and L.N. Lipatov, Yad. Fiz. 15781 (1972) [Sov. J. Nucl. Phys. 15438 (1972)]; G. Altarelli and G. Parisi, Nucl. Phys. B126 298 (1977); Yu.L. Dokshitzer, Zh. Eksp. Teor. Fiz. 731216 (1977) [Sov. Phys. JETP 46641 (1977)].

[3] J. Binnewies, B.A. Kniehl and G. Kramer, Z. Phys. C65 471 (1995); Phys. Rev. D52 4947 (1995); Phys. Rev. D53 3573 (1996).

[4] B.A. Kniehl, G. Kramer and B. Pötter, Nucl. Phys. B582 514 (2000); Phys. Rev. Lett. 855288 (2000); Nucl. Phys. B597 337 (2001).

[5] S. Albino, B.A. Kniehl and G. Kramer, Nucl. Phys. B725 181 (2005); Nucl. Phys. B734 50 (2006).

[6] S. Albino, B.A. Kniehl and G. Kramer, Report No. DESY 08-058, arXiv:0803.2768 [hep-ph] (2008), Nucl. Phys. B (in press).

[7] M. Hirai, S. Kumano, T.H. Nagai and K. Sudoh, Phys. Rev. D75 094009 (2007).

[8] D. de Florian, M. Stratmann and W. Vogelsang, Phys. Rev. D57 5811 (1998); D. de Florian, R. Sassot and M. Stratmann Phys. Rev. D75 114010 (2007); Phys. Rev. D76 074033 (2007).

[9] S. Albino, B.A. Kniehl, G. Kramer and W. Ochs, Phys. Rev. D73 054020 (2006).

[10] H. Aihara et al. (TPC/Two-Gamma Collaboration), Phys. Rev. Lett. 611263 (1988).

[11] R. Itoh et al. (TOPAZ Collaboration), Phys. Lett. B345 335 (1995).

[12] M. Cacciari and S. Catani, Nucl. Phys. B617 253 (2001).

[13] S. Albino, B.A. Kniehl and G. Kramer, Phys. Rev. Lett. 100192002 (2008).

[14] M. Althoff et al. (TASSO Collaboration), Z. Phys. C17 5 (1983).

[15] R. Akers et al. OPAL (Collaboration), Z. Phys. C63 181 (1994).

[16] S.S. Adler et al. (PHENIX Collaboration), Phys. Rev. Lett. 91241803 (2003); J. Adams et al. (STAR Collaboration), Phys. Lett. B637 161 (2006); Phys. Rev. Lett. 97152302 (2006); B.I. Abelev et al. (STAR Collaboration), Phys. Rev. C75 064901 (2007); I. Arsene et al. (BRAHMS Collaboration), Phys. Rev. Lett. 98252001 (2007).

[17] D.E. Acosta et al. (CDF Collaboration), Phys. Rev. D72 052001 (2005). 